\title{
ARTICLE
}

Received 25 Oct 2014 | Accepted 22 Jan 2015 | Published 19 Feb 2015

DOI: $10.1038 /$ ncomms 7360

OPEN

\section{Origin and consequences of silicate glass passivation by surface layers}

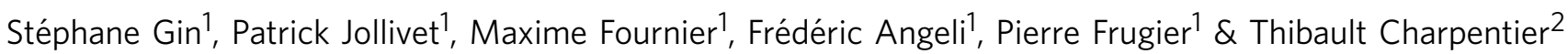

Silicate glasses are durable materials, but are they sufficiently durable to confine highly radioactive wastes for hundreds of thousands years? Addressing this question requires a thorough understanding of the mechanisms underpinning aqueous corrosion of these materials. Here we show that in silica-saturated solution, a model glass of nuclear interest corrodes but at a rate that dramatically drops as a passivating layer forms. Water ingress into the glass, leading to the congruent release of mobile elements ( $\mathrm{B}, \mathrm{Na}$ and $\mathrm{Ca}$ ), is followed by in situ repolymerization of the silicate network. This material is at equilibrium with pore and bulk solutions, and acts as a molecular sieve with a cutoff below $1 \mathrm{~nm}$. The low corrosion rate resulting from the formation of this stable passivating layer enables the objective of durability to be met, while progress in the fundamental understanding of corrosion unlocks the potential for optimizing the design of nuclear glass-geological disposal. 
$\mathrm{T}$ he behaviour of silicate glasses over geological timescales raises challenging scientific issues. One of the most prominent topics with a high social impact is the safe management of highly radioactive wastes originating from spent nuclear fuel reprocessing that, among others, depends on our capacity to predict the long-term corrosion rate of borosilicate glass $^{1-3}$. Geochemists face similar fundamental problems with basaltic glass when calculating the chemical mass balance of the oceans ${ }^{4}$ or when attempting to assess potential $\mathrm{CO}_{2}$ sequestration by silicate rocks ${ }^{5}$. Current rate laws still cannot accurately predict the long-term behaviour of synthetic or natural silicate glasses over geological timescales mostly because of the existence of surface layers between pristine glass and bulk solution that both affect the transport of reactive species and change fluid properties at the glass surface compared with that of the bulk ${ }^{6,7}$. Surface layers are made of amorphous and crystalline metastable phases, formed by in situ condensation and precipitation reactions from aqueous species ${ }^{8}$. Glass dissolves following the Ostwald rule of stages, forming a series of alteration products progressively evolving towards thermodynamically stable phases ${ }^{6,9}$. Depending on the glass composition and on the conditions (for example, temperature, $\mathrm{pH}$, solution composition and flow rate), this transformation into stable compounds can vary from hours to millions of years ${ }^{6,10,11}$ !

In this study, glass corrosion mechanisms were investigated from macroscopic to atomistic scale, through isotopically tagged corrosion experiments performed with international simple glass (ISG) - a six-oxide borosilicate glass used as a reference material by the nuclear glass community ${ }^{1}-$ and the use of complementary analytical techniques to provide evidence of the long-term rate-limiting mechanisms. The main experiment was performed with 16 coupons of ISG glass (with isotopes at the natural abundance) altered for 1 year in a static mode at $90^{\circ} \mathrm{C}$, $\mathrm{pH}_{90}{ }^{\circ} \mathrm{C}$, in a solution initially saturated with amorphous ${ }^{29} \mathrm{SiO}_{2}$ (Supplementary Fig. 1). Previous experiments showed that when altered in deionized water the leaching solution of ISG glass eventually reaches the equilibrium with amorphous silica $\left(\mathrm{SiO}_{2 \mathrm{am}}\right)$ but this requires several years ${ }^{12}$. This starting solution thus enables the first transient stages of glass corrosion to be bypassed and focus placed on the processes governing the longterm rate. In parallel, a similar experiment was conducted with glass powder until its complete alteration, in order to examine the structural changes within the silicate network. The choice of $\mathrm{pH} 7$ was motivated by two reasons. First, after similar investigations carried out at $\mathrm{pH} 9$ and 11.5, it was found that results at $\mathrm{pH} 7$ were the most convincing because glass corrodes about four times faster at $\mathrm{pH} 7$ than at $\mathrm{pH} 9$, although the fundamental processes are the same ${ }^{13}$. This led to larger amounts of material available for in-depth characterization. Second, a pH of 7 is typical of many natural waters; for instance, the water in equilibrium with the claystone studied in France for the storage of nuclear wastes is $\mathrm{pH}$ 7.3 at room temperature ${ }^{14}$. Together, these studies improve our understanding of silicate glass corrosion processes, by linking dissolution kinetics and structural modifications following water ingress into the solid. At this $\mathrm{pH}$, the glass is passivated by a selfhealing and poorly hydrated silica layer in which water is trapped in subnanometric pores. Conclusions can be extended at least until $\mathrm{pH}$ 9.5, leading to general recommendations for the safe management of highly radioactive waste glass.

\section{Results}

Glass still corrodes despite silica saturation conditions. Despite the fact that the solution was initially saturated with respect to amorphous silica, the ISG glass still corrodes. This phenomenon occurred under very stable conditions: $\mathrm{pH}$ was kept constant
(Fig. 1a) and the Si concentration did not change for the entire duration of the test (Fig. 1b). In these conditions, the glass dissolution rate, given by the release time of $\mathrm{B}, \mathrm{Na}$ or $\mathrm{Ca}$ (the three elements behave similarly), dramatically diminished during the experiment (Fig. 1c,d). The rate decrease is $\sim 3.7$ orders of magnitude from the first hours $\left(\sim 500 \mathrm{~nm} \mathrm{~d}^{-1}\right.$, which is close to the maximum rate measured far from equilibrium ${ }^{15}$ ), to 363 days (around $0.1 \mathrm{~nm} \mathrm{~d}^{-1}$ ). In the meantime, $\mathrm{Si}, \mathrm{Al}$ and $\mathrm{Zr}$ remained almost undissolved (at 363 days, the normalized loss of $\mathrm{Al}$ and $\mathrm{Zr}$ are 100 and 1,300 times smaller than that of $\mathrm{B}$, respectively) (Supplementary Table 1). In addition, as the ${ }^{29} \mathrm{Si} /{ }^{28} \mathrm{Si}$ ratio remained stable over time, it could be concluded that ${ }^{29} \mathrm{Si}_{(\mathrm{aq})}$ did not - or if so, only weakly -interact with $\mathrm{Si}$ from the glass during corrosion (Fig. 1b). From this, it seems that the three mobile elements behaved independently from the three low-soluble cations constituting the glassy network. This might seem at odds with the most of accepted models that attributed the drop in the corrosion rate to an increasing activity of the dissolved silica ${ }^{16-19}$.

Mobile species display local gradients. Deeper insights into the glass corrosion mechanisms were obtained from a detailed characterization of the altered glass coupons withdrawn at 7,209 and 363 days. For the three samples, the alteration layer displays three sublayers (Fig. 2). From the solution to the pristine glass a gradient area can first be noticed, which $\mathrm{B}, \mathrm{Na}$ and $\mathrm{Ca}$ concentrations dropped similarly. As time-of-flight secondary ion mass spectroscopy (ToF-SIMS) is known to potentially broaden chemical profiles especially in the case of rough or tilted interfaces ${ }^{20}, \mathrm{~B}$ profile was also characterized by transmission electron microscope (TEM) in order to avoid misinterpretation. Energy-filtered TEM (EFTEM) B mapping performed on a focused ion beam cross-section prepared with the 209-day sample confirmed the position and the width of the B profile given by

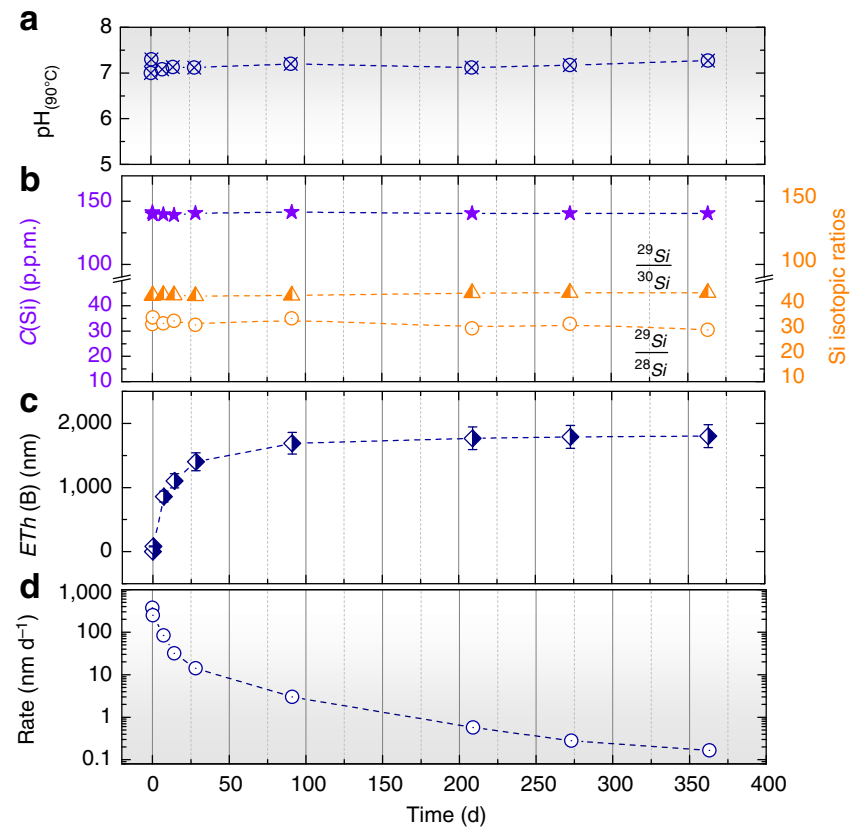

Figure 1 | Data from solution analyses. Time evolution of the solution $\mathrm{pH}$ (a), the concentration of $\mathrm{Si},{ }^{29} \mathrm{Si} /{ }^{28} \mathrm{Si}$ and ${ }^{29} \mathrm{Si} /{ }^{30} \mathrm{Si}$ ratios (b), the equivalent thickness of altered glass (ETh) calculated from $B$ release into solution (c) and the glass dissolution rate (d). The $\mathrm{pH}_{90}{ }^{\circ} \mathrm{C}$ was maintained at $7 \pm 0.25$ by regular additions of diluted $\mathrm{HNO}_{3}$. Boron was used to calculate glass corrosion thickness and rate. This element is known to be a good corrosion tracer as it is very soluble and only slightly retained in the alteration layer (as verified by ToF-SIMS and EFTEM analyses). 


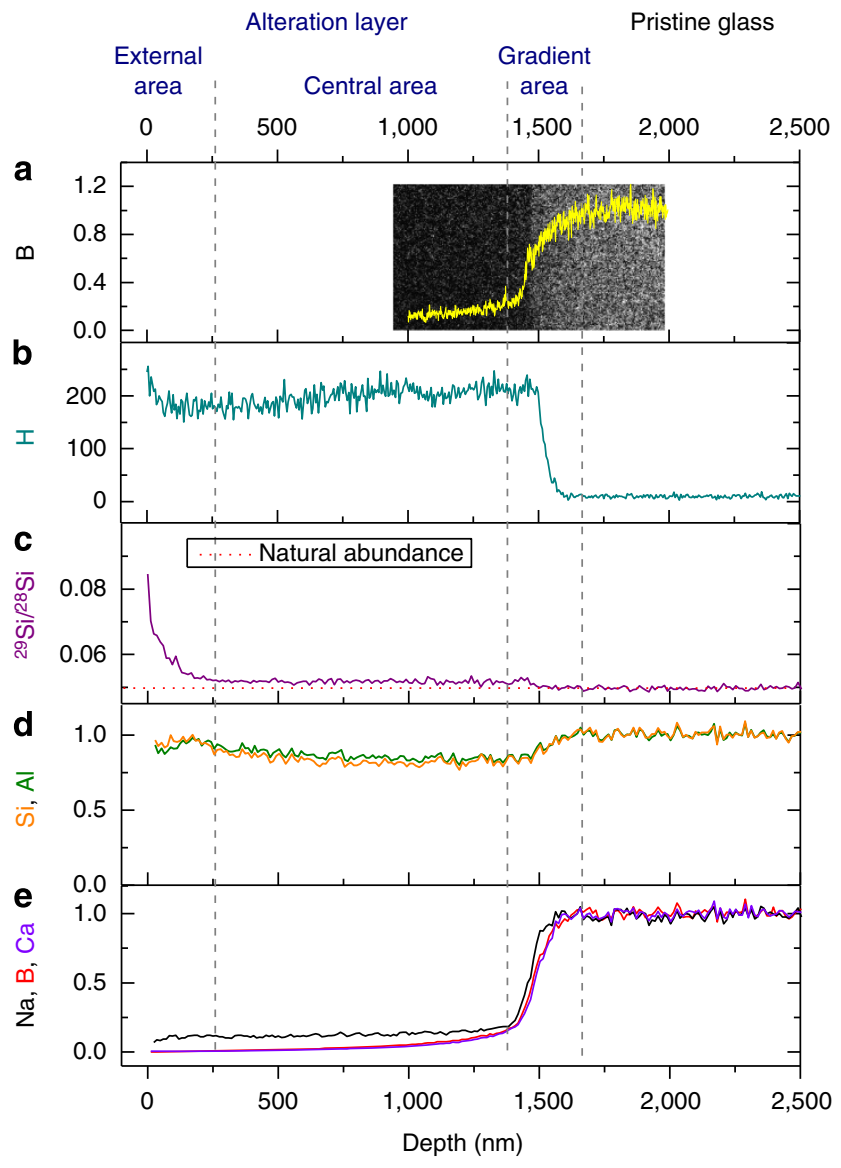

Figure 2 | Elemental profiles within the 209-day sample. (a) EFTEM mapping and the superimposed profile of $B$ concentration (normalized to that of the pristine glass). (b) The $\mathrm{H}$ profile (not-normalized) displaying an anticorrelated steeper gradient than that of the other mobile species of the glass (B, $\mathrm{Ca}$ and $\mathrm{Na}$ ). (c) ${ }^{29} \mathrm{Si} /{ }^{28} \mathrm{Si}$ ratio exhibiting a slight enrichment in the first $250 \mathrm{~nm}$ of the alteration layer (this depth is possibly overestimated according to the caveat given in Methods) and then a constant value a few percent above the natural abundance $(1( \pm 1) \%$ at 7 days, $5( \pm 1) \%$ at 209 days and $4( \pm 1) \%$ at 363 days). This ratio has to be compared with that of the bulk solution $(\sim 30)$. (d,e) Normalized profiles of sparingly soluble glass formers ( $\mathrm{Si}$ and $\mathrm{Al}$ ) and soluble elements $(\mathrm{Na}, \mathrm{Ca}$ and $\mathrm{B}$ ). Si and $\mathrm{Al}$ remain at a near constant concentration in the alteration layer, whereas mobile species $(\mathrm{B}, \mathrm{Ca}$ and $\mathrm{Na}$ ) are leached out of the layer except near the reaction front, where a gradient is clearly visible. Note that $\mathrm{K}$ is highly present in the alteration layer, but it is not displayed because its signal was saturated.

ToF-SIMS (Fig. 2a). ToF-SIMS also revealed that B, Na and Ca gradients are well anticorrelated with that of $\mathrm{H}$ (Fig. $2 \mathrm{~b}$ and Supplementary Fig. 2). This pattern is generally attributed to ion exchange between exogenous positively charged species $\left(\mathrm{H}^{+}\right.$, $\mathrm{H}_{3} \mathrm{O}^{+}$and $\mathrm{K}^{+}$) and $\mathrm{Na}^{+}, \mathrm{Ca}^{++}$from the glass and accompanied by the fast hydrolysis of $\mathrm{B}-\mathrm{O}$ bonds ${ }^{21,22}$. Beyond this gradient area, B and Ca concentrations slowly decreased to zero, whereas $\mathrm{Na}$ remained at a low but constant concentration, likely to act as a charge compensator along with $\mathrm{K}$ for fourfold coordinated $\mathrm{Al}$ species and sixfold coordinated $\mathrm{Zr}$ species ${ }^{23}$. This large, central area showed a nearly flat concentration of all the glass constituents. Finally, an external area is visible, mainly characterized by significant changes in $\mathrm{Si}$ isotopic ratios (Fig. 2c).

The alteration layer is not a precipitate. In the central area region the ${ }^{29} \mathrm{Si} /{ }^{28} \mathrm{Si}$ ratio plateaus at a few percent above the

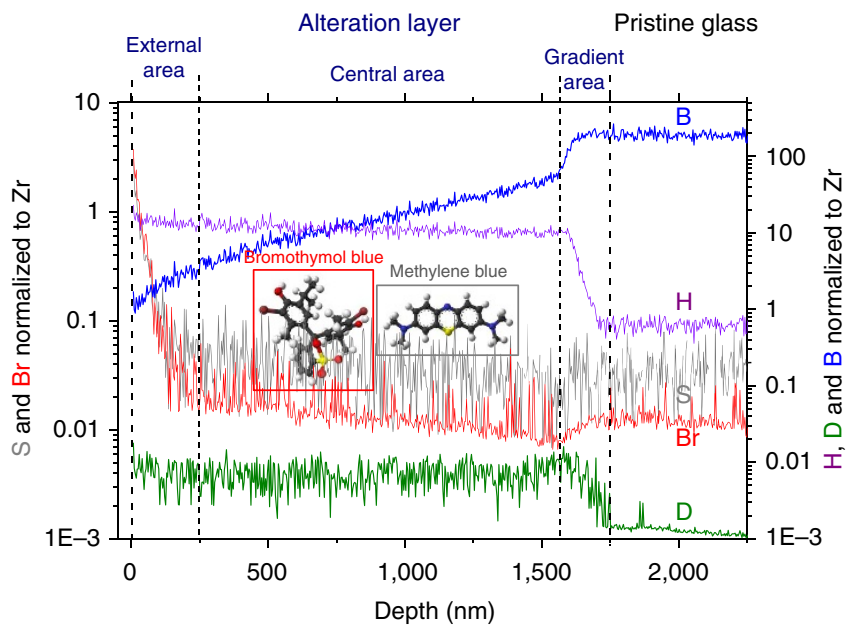

Figure 3 | Elemental profiles within the 209-days sample after the tracing experiment with $\mathbf{D}_{\mathbf{2}} \mathbf{O}$ and dyes. The sample spent $100 \mathrm{~h}$ at room temperature in contact with these molecules before ToF-SIMS depth profiling. $S, B$ and $D$ are proxies for methylene blue ( $S$ in grey), bromothymol blue ( $\mathrm{Br}$ in red) and $\mathrm{D}_{2} \mathrm{O}$ ( $\mathrm{D}$ in green) molecules, respectively. $\mathrm{B}$ and $\mathrm{H}$ are displayed as reminders of the location of the different sublayers.

natural abundance (Fig. 2c). This slight enrichment of Si species supplied by the solution increased and stayed constant between 209 and 363 days. At the steady state, it represents one atom of Si supplied by the solution per 600 coming from the glass. This result suggests that the central part of the alteration layer is not a diffusion barrier for $\mathrm{Si}_{(\mathrm{aq})}$ as diffusion-limiting process would have led to chemical gradients. Therefore, one can assume that a thermodynamic equilibrium between the pore solution and the silicate network was achieved. The external area, $\sim 250-\mathrm{nm}$ thick (or less, according to the caveat given in the Methods section) showed a significant increase in the ${ }^{29} \mathrm{Si} /{ }^{28} \mathrm{Si}$, especially in the first nanometre (Supplementary Fig. 3) where the isotopic ratio goes up to 1 . Moreover, the profiles of the outermost sublayer are similar at 7 and 209 days (Supplementary Fig. 3). The latter observations strongly suggest that the glass surface underwent dissolution and reprecipitation reactions, and that an equilibrium between the external surface of the glass and the bulk solution was achieved within a few days. Overall, these findings are consistent with those obtained in a far more diluted medium, favouring the hydrolysis of $\mathrm{Si}-\mathrm{O}-\mathrm{M}$ bonds $(\mathrm{M}=\mathrm{Si}, \mathrm{Al}$ and $\mathrm{Zr})$, where the whole alteration layer was significantly and gradually enriched in Si supplied by the solution ${ }^{24}$. Here except at the extreme surface, almost none of the silicon atoms of the glassy network were completely hydrolysed. These observations lead to two conclusions: first, a thermodynamic equilibrium is achieved between the surface of the altered glass and the bulk solution despite the absence of isotopic equilibrium inside the porous material; second, the altered glass cannot result from the congruent dissolution of the glass. The properties of the alteration layer next needed to be determined, to better understand the behaviour of the mobile species.

The alteration layer acts as a molecular sieve. It has long been thought that Si-rich amorphous alteration layers formed during glass or mineral corrosion could be passivating ${ }^{25-28}$. However, the location and the mechanism limiting the transport of reactive species remained unclear. A post-experiment tracing test carried out with the 209-day glass sample showed that exogenous molecules added to solution at the end of the experiment behaved differently depending on their size (Fig. 3). Deuterium $(0.27 \mathrm{~nm}$ 
in diameter) diffused easily up to the gradient area, and then its concentration dropped similarly to that of $\mathrm{H}$, whereas the two dye molecules ( $1 \mathrm{~nm}$ in diameter) could only enter the first $250 \mathrm{~nm}$ of the alteration layer. As a result, the central part of the alteration layer acted as a molecular sieve with a cutoff $<1 \mathrm{~nm}$. According to the literature, such narrow channels have a huge impact on the fluid properties within the alteration layer ${ }^{29}$ : the density, dielectric constant, activity and diffusivity of water are expected to significantly differ from that of the bulk, as well as phase solubility and ion hydration.

The growth of the alteration layer affects the transport of aqueous species. The B profiles determined by ToF-SIMS (Fig. 4) display complex shapes that cannot be explained by classical interdiffusion models (Models 1 and 2 in our case) ${ }^{30-32}$. Although Model 2 fits better with our experimental data than Model 1, it must be considered that the diffusion coefficients of water and $\mathrm{B}$ diminish with time. Moreover, the shape of the $\mathrm{B}$ profiles is not well fitted with Model 2. However, simple considerations can be taken into account. The initial diffusion coefficient of mobile species of the glass through the newly formed alteration layer, $D_{\max }$, was derived from the first day of alteration of the 4- to $6-\mu \mathrm{m}$ glass powder placed in the same conditions as the glass monoliths and studied by solid-state nuclear magnetic resonance (NMR) spectroscopy. According to the corresponding value of $D_{\max }$ of $6 \times 10^{-19} \mathrm{~m}^{2} \mathrm{~s}-1$, a glass
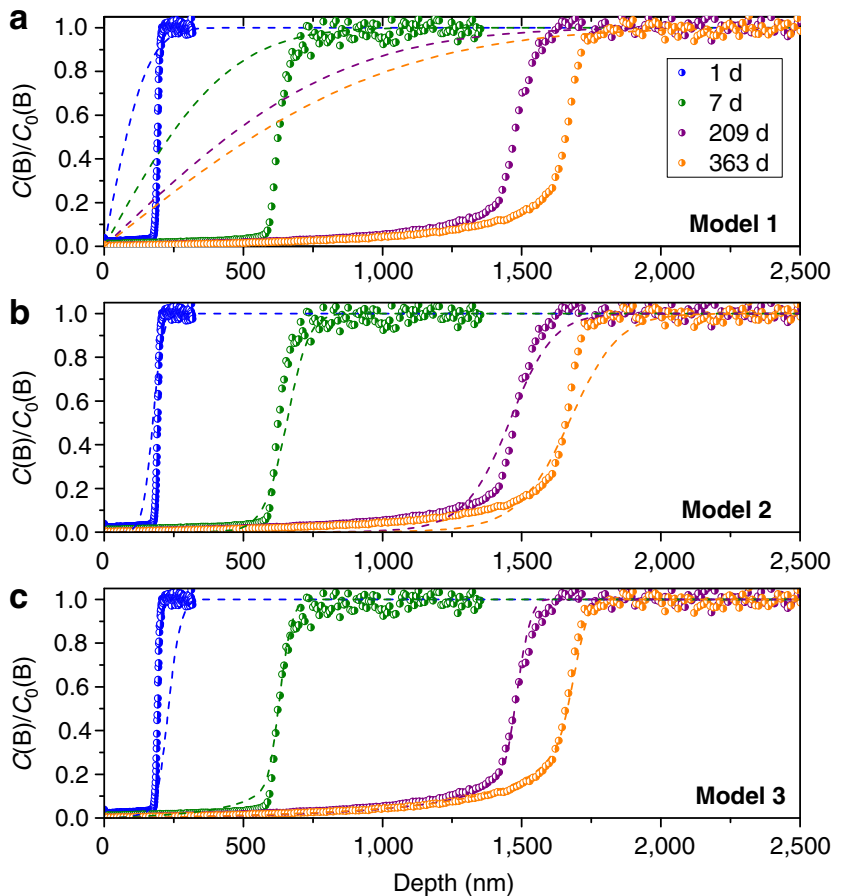

Figure 4 | Time evolution of boron profile. Normalized B concentrations obtained with ToF-SIMS depth profiling (circles) are compared with simulations (dashed lines). The widths of the gradient area are $25 \mathrm{~nm}$ at the first duration, $150 \mathrm{~nm}$ at 7 days and $200 \mathrm{~nm}$ at the two last durations. (a) Comparison with Model 1, the simplest interdiffusion model, assuming a semi-infinite medium with a constant diffusion coefficient $D$ of $6 \times 10^{-19} \mathrm{~m}^{2} \mathrm{~s}^{-1}$. (b) Comparison with Model 2, a refined interdiffusion model assuming that water diffuses more slowly than mobile species from the glass. Here $b=40,000$ and $D_{w}=10^{-20}, 2 \times 10^{-20}, 3.3 \times 10^{-21}$ and $2.5 \times 10^{-21} \mathrm{~m}^{2} \mathrm{~s}^{-1}$ at $1,7,209$ and 363 days, respectively. (c) Comparison with Model 3 , an advanced model with $D$ spatially depending on the water concentration. Fits were obtained with $D_{\max }=7.6( \pm 4.3) 10^{-19} \mathrm{~m}^{2} \mathrm{~s}^{-1}$, $\alpha=1.87( \pm 0.23), \beta=4.63( \pm 0.48)$. alteration thickness of $5.3 \mu \mathrm{m}$ after 363 days was expected, a value three times greater than the actual thickness. This result confirms the hypothesis derived from Model 2, suggesting that the apparent diffusivity of mobile species became lower than at the beginning. To explain this effect, a third model was developed (Model 3). Following this model (Fig. 4c), D is expected to drop by $4-5$ orders of magnitude between the outermost layer and the reaction front, to account for the peculiar shape of the B profiles. The reasonable good quality of the fit provided by this model, with low variation of the fitting parameters, suggests that the transport of mobile species is space-dependent explaining why it was suggested to be time-dependent. $D$ could thus be positively correlated to a local concentration of the chemically rate-limiting species, probably one of the water species. A strong hypothesis to account for this phenomenon, although never investigated in silica-saturated conditions, is the reorganization of the silicate network following the departure of the mobile species ${ }^{22,33,34}$. This is the aim of the following investigations.

Silica does not polymerize in solution. Although $\mathrm{Si}_{(\mathrm{aq})}$ is poorly reactive with corroding glass, we investigated both its speciation in solution and the structure of the alteration layer by liquid-state and solid-state ${ }^{29} \mathrm{Si}$ NMR spectroscopy. Figure 5a shows only one species of $\mathrm{Si}_{(\mathrm{aq})}$ in the initial solution and in the leachate, which corresponds to monomers $\left(\mathrm{H}_{4} \mathrm{SiO}_{4}\right)^{35}$. Therefore, polymerization reactions that could be expected ${ }^{36}$ in these highly concentrated solutions did not occur. As a result, it can be supposed that $\operatorname{Si}_{(\mathrm{aq})}$ species are small enough $(\sim 0.5 \mathrm{~nm})$ to freely diffuse within the alteration layer up to the gradient area. This assumption is in agreement with the slight homogeneous increase in the ${ }^{29} \mathrm{Si} /{ }^{28} \mathrm{Si}$ ratio in the central area of the altered layer (Fig. 2c).
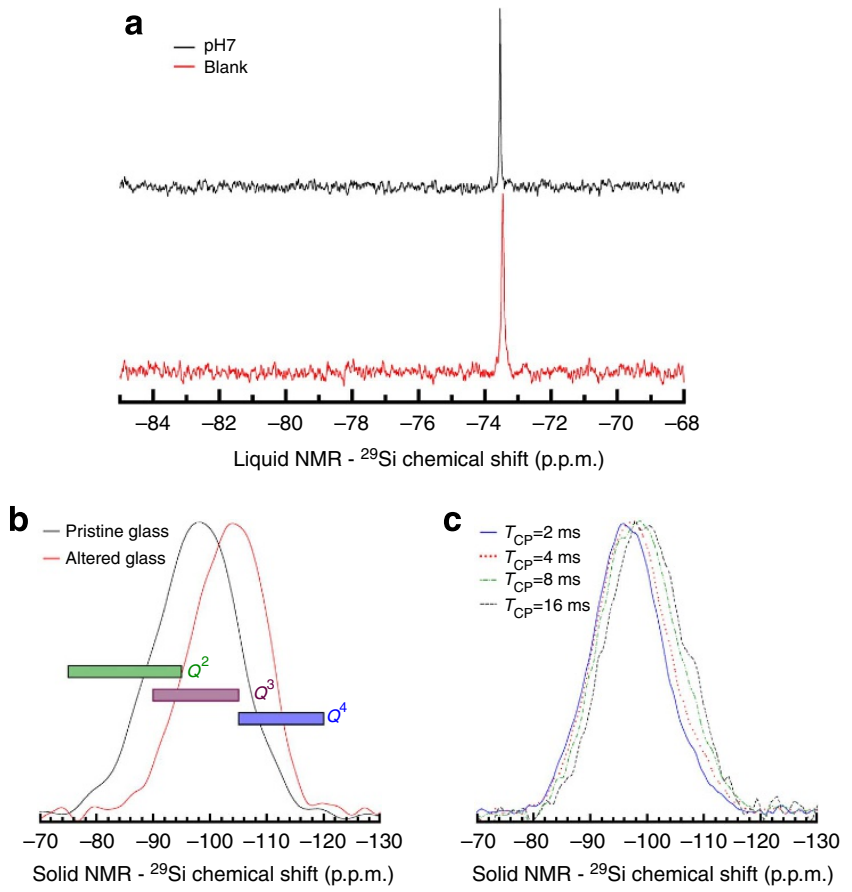

Figure 5 | Liquid-state and solid-state ${ }^{29}$ Si NMR spectroscopy performed both on solution and on pristine and altered glass. (a) Leaching solution at the end of glass alteration $(\mathrm{pH} 7)$ compared with the initial ${ }^{29}$ Si-enriched solution (Blank). (b) Pristine and fully altered glass from MAS NMR and (c) fully altered glass from CP-MAS NMR, obtained with various proton-tosilicon magnetization transfer times $\left(T_{C P}\right)$ from 2 to $16 \mathrm{~ms}$. $Q^{n}$ refers to the degree of connectivity of a $\mathrm{SiO}_{2}$ tetrahedron, where $n=0-4$ corresponds to the number of bridging oxygen atoms. 
In situ reorganization after silicate network corrosion.. Solidstate ${ }^{29}$ Si MAS NMR spectroscopy was performed on fully altered grains of glass corroded under similar conditions as the monoliths. Owing to their different electronegativity, the main glassformer cations $\mathrm{Al},{ }^{\mathrm{IV}} \mathrm{B}$ and $\mathrm{Zr}$ increase the chemical shift, $\delta_{\mathrm{Si}}$ (refs 37-40), whereas Ca gives an opposite effect because of its location near the nonbridging oxygen atoms ${ }^{41}$. The Carr Purcell Meiboom Gill (CPMG) sequence ${ }^{42}$ as displayed in Fig. $5 \mathrm{~b}$ indicates a negative shift of $\delta_{\mathrm{Si}}$ from pristine to altered glass. In a first approximation, this shift could be an artifact tied to the release of B. However, because of the opposite effect of $\mathrm{Ca}$, and the low proportion ${ }^{\mathrm{IV}} \mathrm{B}$ compared with $\mathrm{Si}$, the observed shift is more likely to be attributed to a higher degree of polymerization of the alteration layer compared with the glass. This hypothesis is confirmed by cross-polarization ${ }^{1} \mathrm{H}^{2}{ }^{29} \mathrm{Si}$ NMR analyses (Fig. 5 c). By increasing the magnetization transfer time, larger distances between protons and $\mathrm{Si}$ were probed. Excitations of species with high numbers of bridging atoms to $\mathrm{Si}\left(\mathrm{SiO}_{4}\right)$ are then at the origin of the $\delta_{\mathrm{Si}}$ shifts to lower values. To our knowledge, this is the first direct evidence of the reorganization of the silicate network at high reaction progress. This reorganization was not expected based on the chemical analyses of the $\mathrm{Si}$ behaviour both in solution and in the altered glass. From a structural point of view, it may be in agreement with Molecular Dynamic and Monte Carlo simulations, which showed that the hydrolysis of $\mathrm{B}-\mathrm{O}-\mathrm{Si}$ bonds, the release of $\mathrm{Na}$ and $\mathrm{Ca}$ from nonbridging oxygen atom, and the potential hydrolysis of some $\mathrm{Si}-\mathrm{O}-\mathrm{Si}$ bonds first form $\mathrm{Si}$ $\mathrm{OH}$ groups that can then condense to reform $\mathrm{Si}-\mathrm{O}-\mathrm{Si}$ bonds ${ }^{21}$. This in situ reorganization is likely to be less energy-consuming than the complete hydrolysis of the glass species and their precipitation into more stable phases.

\section{Discussion}

Two visions of the rate-limiting mechanisms are currently under discussion in the literature. On the one hand, glass is expected to dissolve congruently in a nanometre-size film of water, and dissolved species precipitate from the liquid phase ${ }^{43-46}$. On the other hand, glass undergoes ion exchange, whereby positively charged water species replace alkalis in glass-modifier position with partial or no dissolution of the remaining silicate network once silica saturation is achieved in the bulk solution ${ }^{18,22,47}$. Our findings show that none of these paradigms are strictly correct at least for explaining the rate under silica saturation conditions. The release of mobile cations from the glass is associated with a high repolymerization of the silicate network leading to a selfreorganized submicroporous material, in which only small molecules $(<1 \mathrm{~nm})$ can diffuse. Preliminary data suggest that the water content of this material is very low $(\sim 3 \mathrm{wt} \%)$ compared with porous gels formed far from saturation ( 10-20 wt\%). Moreover, the most of this material is passivating, as evidenced by a significant decrease in the mobile species diffusivity. The removal of $\mathrm{B}, \mathrm{Na}$ and $\mathrm{Ca}$ from the glass deeply affects the structure and the properties of the alteration layer, triggering the repolymerization of the silicate network and the resulting material in turn affects the release of mobile species. In addition, it could be noted that the porosity clogging of the external part of the alteration layer, as demonstrated in other studies ${ }^{48,49}$, is not a prerequisite for the rate drop. It happens for some glasses, making them more durable, but in most cases the porosity stays open as shown here and the passivation occurs with the growth of the self-reorganized alteration layer 9 . The reason why $D$ drops, making the glass/alteration layer interphase more efficient than the glass itself in preventing water ingress, may now be discussed. On the basis of thermodynamic and kinetic considerations, at least three hypotheses seem possible. (1) The water mobility and reactivity within the alteration layer are lower than in the bulk solution. This hypothesis is supported by the extremely small pore size of the alteration layer $(<1 \mathrm{~nm})$ accounting for the fact that there are no free water molecules in this medium ${ }^{50}$. (2) The flux of water towards the glass drops along with the growth of the alteration layer. (3) Liquid and solid phases within the reacting interphase approach a global thermodynamic equilibrium. Model 3, successfully applied to our experimental results, cannot help rank the previous hypotheses. Nonetheless, the results strongly suggest that transport of elements and chemical reactivity of nanoconfined water are closely linked ${ }^{51}$. It is worth noting that the diffusivity of $\mathrm{He}-\mathrm{a}$ chemically inert atom with the same size as a water molecule-in this type of glass is $\sim 10^{-15} \mathrm{~m}^{2} \mathrm{~s}-1$ at $90^{\circ} \mathrm{C}$ (ref. 52), a value 4-9 orders of magnitude lower than water diffusivity in pristine and altered glass. This huge difference supports the idea that water mobility in pristine and altered glass is strongly affected by chemical interactions with the solid phase. These reactions need to be better understood.

These findings give rise to several points. First of all, glass corrosion mechanisms-especially those controlling the longterm behaviour of glass in confined medium-are better understood. As the literature reports strong mechanistic analogies between nuclear and natural glasses ${ }^{53}$, the long-term behaviour of basaltic glass in confined environments could be re-evaluated. For the first time, it has been demonstrated that under silica saturation conditions, (i) the reorganized alteration layer achieves equilibrium with the bulk and pore solutions and (ii) the residual corrosion rate dramatically diminishes due to transport-limiting effects near the glass surface. On the conservative assumption that boron release rate stops diminishing, the time required to corrode $1 \mathrm{~cm}$ of glass at a rate of $0.1 \mathrm{~nm} \mathrm{~d}^{-1}$ (the rate obtained at 363 days, Fig. 1d) would exceeds 250,000 years at $90^{\circ} \mathrm{C}$. It is worth noting that most of the results presented in this paper have been confirmed at $\mathrm{pH} \mathrm{9,}$ although glass corrosion is slower and the resulting layers are more difficult to characterize because they are thinner. Hence, such conditions seem favourable to guarantee a very high durability of nuclear glass. According to our results and the large experimental database on nuclear glass corrosion, including industrial materials, these conditions could be extended to the following: $T \leq 90^{\circ} \mathrm{C}, 7 \leq \mathrm{pH} \leq 9.5$, silica-saturated solution and low renewal rate of the solution. The disposal of glass packages that are under consideration in many countries (for example, the USA, the UK, France, Japan, Belgium and Germany) could be optimized by meeting such conditions. It must, however, be kept in mind that other reactions not observed here can make the glass less durable. Among them, the most detrimental effect is the precipitation of silicate minerals (phyllosilicates, iron-silicates, zeolites, calcium-silicate-hydrates, and so on) that sustain glass corrosion by consuming $\mathrm{Si}_{(\mathrm{aq})}$ and potentially other glass formers from both the solution and the alteration layer and disrupting the passivating layer ${ }^{6,54-56}$. The mechanism is well known but only a few studies have attempted to model the competition between the amorphous layer and other minerals owing to the lack of knowledge on this nonstoichiometric material (composition, dissolution rate and solubility $)^{57,58}$. Thanks to the methodology and the protocols proposed here, our study opens the way to further investigations and applications.

\section{Methods}

Glass preparation. The ISG glass $\left(60.2 \mathrm{SiO}_{2}, 16.0 \mathrm{~B}_{2} \mathrm{O}_{3}, 12.6 \mathrm{Na}_{2} \mathrm{O}, 5.7 \mathrm{CaO}, 3.8\right.$ $\mathrm{Al}_{2} \mathrm{O}_{3}$ and $1.7 \mathrm{ZrO}_{2}$ in mol\%) was produced in 2012 by MoSci Corporation (Rolla, MO, USA). The glass was melted in platinum-rhodium crucibles in an electric furnace at $1,300^{\circ} \mathrm{C}$ for $\sim 4 \mathrm{~h}$, stirred once with a quartz rod and cast into a graphite mold. The ingots were annealed at $569^{\circ} \mathrm{C}$ for $6 \mathrm{~h}$ in an electric oven and cooled to 
room temperature at a rate of $50{ }^{\circ} \mathrm{C}$ per hour. Sixteen coupons of $2 \times 2 \times 0.11 \mathrm{~cm}^{3}$ each with only one large face polished were put in polytetrafluoroethylene holders and placed at $90^{\circ} \mathrm{C}$ in a perfluoroalkoxy vessel containing $380 \mathrm{ml}$ of solution initially saturated with amorphous ${ }^{29} \mathrm{SiO}_{2}\left(C(\mathrm{Si})=141 \mathrm{mg} \cdot \mathrm{l}^{-1}\right.$; ;upplementary Fig. 1). Note that glass coupons were prepared with one face polished and the other let raw after being cut with a diamond saw in order to see how fast rough surfaces become smooth due to corrosion. This question is partly addressed in a similar study conducted at $\mathrm{pH} 11.5$ (ref. 13). To obtain the starting solution, ${ }^{29} \mathrm{SiO}_{2}$ (Eurisotop, ${ }^{29} \mathrm{Si}>95 \%$ ) was mixed with $\mathrm{KOH}$ (Suprapur) and melted at $600{ }^{\circ} \mathrm{C}$. The resulting potassium silicate was then dissolved in deionized water. This protocol led to a concentration of $\mathrm{K}_{\text {of }} 6.8 \mathrm{gl}^{-1}$. It was verified separately that the concentration of $\mathrm{K}$ has no influence on the glass corrosion behaviour. The $\mathrm{pH}_{90}{ }^{\circ} \mathrm{C}$ was maintained at $7 \pm 0.25$ throughout the experiment with regular additions of $\mathrm{HNO}_{3} 10^{-2} \mathrm{M}$. The reactive surface area of a glass coupon was estimated at $15 \pm 2 \mathrm{~cm}^{2}$ by measuring the 'not-normalized' initial dissolution rate at $\mathrm{pH} 9$ (in $\mathrm{gd}^{-1}$ ) of a glass coupon similar to those utilized in this study and then dividing this rate by the normalized dissolution rate (in $\mathrm{g} \mathrm{m}^{-2} \mathrm{~d}^{-1}$ ) obtained in the same conditions with a fully polished coupon. More details are given in ref. 13.

For the solid-state NMR analysis, $300 \mathrm{mg}$ of small 4 - to 6 - $\mu \mathrm{m}$-diameter particles of the same glass were prepared by milling and decantation in acetone. This powder was altered under similar conditions $\left(90^{\circ} \mathrm{C}, \mathrm{pH}_{90^{\circ} \mathrm{C}} 7, \mathrm{C}(\mathrm{Si})=135 \mathrm{mgl}^{-1}\right)$ until the complete release of $B$.

Solution analysis. The solution was regularly sampled and cations were analysed by inductively coupled plasma-optical emission spectroscopy (Thermo Scientific, ICAP 6300 DUO). Silicon isotopes were analysed by multicollector inductively coupled plasma-mass spectroscopy (Nu Instruments Nu Plasma HR). The equivalent thickness of altered glass, ETh, was calculated from B concentration at a sampling duration $t$ as follows:

$$
E \operatorname{Th}(\mathrm{B})_{t}=E \operatorname{Th}(\mathrm{B})_{t-1}+\frac{\left(C(\mathrm{~B})_{t}-C(\mathrm{~B})_{t-1}\right) V_{t}}{\rho \times N_{t} \times S_{0} \times x_{\mathrm{B}}}
$$

where $C(\mathrm{~B})$ is the $\mathrm{B}$ concentration, $V_{t}$ the solution volume at the time $t, \rho$ the glass density $\left(2.50 \mathrm{~g} \mathrm{~cm}^{-3}\right), N_{t}$ the number of glass coupons still present in the vessel at the time $t, S_{0}$ the reactive surface area of a glass coupon $\left(15 \pm 2 \mathrm{~cm}^{2}\right)$ and $x_{\mathrm{B}}$ the mass fraction of $\mathrm{B}$ in the glass (0.0539). The glass dissolution rate $r$ is given by:

$$
r=\frac{d(E T h(\mathrm{~B}))}{d t}
$$

The uncertainties for $E T h(\mathrm{~B})$ and $r$ are $10 \%$ and $30 \%$, respectively.

Post-experiment tracing test. At three durations (7, 209 and 363 days) a glass coupon was withdrawn, rinsed and cut into four pieces before characterization. One piece of the 209-day sample was immersed $100 \mathrm{~h}$ at room temperature in a solution at $\mathrm{pH}$ 7, saturated with $\mathrm{SiO}_{2 \mathrm{am}}$ and spiked with $1.2 \mathrm{moll}^{-1} \mathrm{D}_{2} \mathrm{O}, 8.0$ $10^{-5} \mathrm{~mol}^{-1}$ bromothymol blue and $4.410^{-4} \mathrm{moll}^{-1}$ methylene blue.

Depth profiling analyses. The 209-day glass coupon before and after immersion in the spiked solution, along with the two coupons collected on 7 and 363 days and a last one $\left(0.5 \times 0.5 \times 0.2 \mathrm{~cm}^{3}\right)$ added to the solution at the end of the experiment and withdrawn 1 day after, were analysed by ToF-SIMS (IONTOF TOF 5). Two sputtering beams, analysing a surface area of $\sim 60 \times 60 \mu \mathrm{m}^{2}$, were utilized: $\mathrm{Cs}^{+}$ (for $\mathrm{D}, \mathrm{H}, \mathrm{Br}$ and S) or $\mathrm{O}_{2}^{+}$(for the other elements). The oxygen beam was either set at $2 \mathrm{keV}, 545 \mathrm{nA}$ to quickly analyse the whole alteration layer and some pristine glass, or at $500 \mathrm{eV}, 100 \mathrm{nA}$ to get more accurate data of the external part of the alteration layer. It must be noted that the first analytical conditions provide a lower depth resolution $(\sim 12$ versus $\sim 0.1 \mathrm{~nm}$ per step $)$ and also tend to shift the profiles of species weakly bonded to the network because of energetic collisions with $\mathrm{O}_{2}^{+}$ ions (the case of ${ }^{29} \mathrm{Si}$ atoms, which have diffused from the bulk solution, as well as $\mathrm{Br}$ and S). Elemental profiles were normalized to that of $\mathrm{Zr}$ to avoid matrix effects. For the glass components $i(i=\mathrm{Si}, \mathrm{B}, \mathrm{Na}, \mathrm{Al}, \mathrm{Ca})$, the ratios $i / \mathrm{Zr}$ were then normalized to that measured in the pristine glass.

Modelling B profiles. Boron profiles were fitted according to three models. Model 1 assumes that the glass is a nonreactive, semi-infinite medium with a planar, nonstationary (that is, retreating) fluid-solid interface. The normalized concentration of $\mathrm{B}$, resulting from the resolution of the second Fick's law, is given by ${ }^{59}$ :

$$
\frac{C(\mathrm{~B})}{C_{0}(\mathrm{~B})}=\operatorname{erf}\left(\frac{z}{2 \sqrt{D t}}\right)
$$

where $C(\mathrm{~B})$ and $C_{0}(\mathrm{~B})$ are the concentrations of $\mathrm{B}$ at a given depth $z$ and in the pristine glass, respectively, $D$ is the apparent diffusion of $B-$ a constant parameter in this model-and $t$ the time.
Model 2 is based on Doremus' model assuming that water diffuses more slowly than $\mathrm{B}^{32}$. The normalized concentration of $\mathrm{B}$ becomes:

$$
\frac{C(\mathrm{~B})}{C_{0}(\mathrm{~B})}=\frac{1-\operatorname{erfc}\left(\frac{z}{2 \sqrt{D_{w t}}}\right)}{1+b \times \operatorname{erfc}\left(\frac{z}{2 \sqrt{D_{w} t}}\right)}
$$

where $D_{\mathrm{w}}$ is the diffusion coefficient of water, $b=D / D_{\mathrm{w}}-1$.

Unlike Model 2, Model 3 assumes that $D$ depends on local concentration of water, a likely rate-limiting species. As water replaces mobile species released by the glass, it is assumed in a first approximation that the concentration of water is inversely proportional to the B concentration. Equation 4 is postulated to account for $D$ variations with local boron concentrations.

$$
\log _{10} \frac{D}{D_{\max }}=-\beta\left(\frac{C(\mathrm{~B})}{C_{0}(\mathrm{~B})}\right)^{\frac{1}{\alpha}}
$$

where $D_{\max }$ corresponds to the maximal value of the apparent diffusion of B in the alteration layer, while $\alpha$ and $\beta$ are two fitting parameters. For $\beta=1, D$ is 10 times lower than $D_{\max }, 100$ times in case $\beta=2$. Parameter $\alpha$ describes how $D$ drops with increasing B concentration (decreasing water concentration). The value of $D_{\max }$ $\left(6 \times 10^{-19} \mathrm{~m}^{2} \mathrm{~s}^{-1}\right)$ was approximated by measuring the release of mobile species during the first stage of glass corrosion, when preparing the powder for the NMR study. The second Fick's law, in which $D$ variations are given by Equation 5 was solved numerically following a finite element approach.

Electronic microscopy. Scanning electron microscope (SEM) observations as well as TEM analyses (energy-dispersive X-ray spectroscopy and EFTEM) were also performed on the 209-day sample to confirm our results. For the TEM study, a thin film of $\sim 100$-nm thick was prepared with a dual beam SEM/FIB microscope (FEI Helios NanoLab 600) following the method described in ref. 60. EFTEM boron images were acquired with a TEM FEI Titan 80-300 Cs operating at $200 \mathrm{kV}$ under the following conditions: acquisition time $10 \mathrm{~s}$ per image, energy window width $20 \mathrm{eV}$ and a bining of $2 \times 2$, giving $1,024 \times 1,024$ pixel images. Images were processed with Gatan's Digital Micrograph software package. The three-window technique was used to obtain boron elemental maps ${ }^{61}$. The B profile was calculated with the ImageJ software

NMR spectroscopy. Liquid-state ${ }^{29} \mathrm{Si} \mathrm{NMR} \mathrm{spectra} \mathrm{were} \mathrm{recorded} \mathrm{on} \mathrm{a} 400-\mathrm{MHz}$ Agilent DD2 spectrometer equipped with the OneNMR 5-mm probe and without sample-spinning, following the procedure described elsewhere ${ }^{13} \cdot{ }^{29} \mathrm{Si}$ crosspolarization magic-angle spinning (CP-MAS) NMR spectra were collected on a Bruker Avance $300 \mathrm{MHz}$ spectrometer (magnetic field $7.05 \mathrm{~T}$ ) using a 4-mm (rotor outer diameter) Bruker CP-MAS Probe and sample-spinning frequency of $10,000 \mathrm{~Hz}$. CPMG ${ }^{29} \mathrm{Si}$ MAS spectra collected are described in ref. 40 , co-adding typically 20-40 echoes, with a recyle delay of $20 \mathrm{~s}$ (no changes in lineshape were observed between 2 and $200 \mathrm{~s}$ ). CP-MAS ${ }^{29} \mathrm{Si}\left\{{ }^{1} \mathrm{H}\right\}$ spectra were collected with a recycle delay of $2 \mathrm{~s}$ and ${ }^{1} \mathrm{H}_{-}{ }^{29} \mathrm{Si}$ magnetization transfer ranging from 0.5 to $16 \mathrm{~ms}$.

Consistency of the measurements. Consistency of the glass alteration thickness measurements was verified using four independent techniques. It was found that all the measurements are consistent within a range of $\pm 15 \%$ (Supplementary Fig. 4).

\section{References}

1. Gin, S. et al. An international initiative on long-term behavior of high-level nuclear waste glass. Mater. Today 16, 243-248 (2013).

2. Grambow, B. Nuclear waste glasses-how durable? Elements 2, 357-364 (2006).

3. Ojovan, M. I. \& Lee, W. E. Glassy wasteforms for nuclear waste immobilization. Metall. Mater. Trans. A 42A, 837-851 (2011).

4. Walton, A. W., Schiffman, P. \& Macpherson, G. L. Alteration of hyaloclastites in the HSDP 2 Phase 1 Drill Core: 2. Mass balance of the conversion of sideromelane to palagonite and chabazite. Geochem. Geophys. Geosyst. 6, Q09G19 (2005).

5. Sissmann, O. et al. Enhanced olivine carbonation within a basalt as compared to single-phase experiments: reevaluating the potential of $\mathrm{CO} 2$ mineral sequestration. Environ. Sci. Technol. 48, 5512-5519 (2014).

6. Vienna, J. D., Ryan, J. V., Gin, S. \& Inagaki, Y. Current Understanding and remaining challenges in modeling long-term degradation of borosilicate nuclear waste glasses. Int. J. Appl. Glass Sci. 4, 283-294 (2013).

7. Grambow, B. \& Muller, R. First-order dissolution rate law and the role of surface layers in glass performance assessment. J. Nucl. Mater. 298, 112-124 (2001).

8. Matzke, H. \& Vernaz, E. Thermal and physicochemical properties important for the long-term behavior of nuclear waste glasses. J. Nucl. Mater. 201, 295-309 (1993).

9. Gin, S. et al. Nuclear glass durability: new insight into alteration layer properties. J. Phys. Chem. C 115, 18696-18706 (2011). 
10. McGrail, B. P., Ebert, W. L., Bakel, A. J. \& Peeler, D. K. Measurement of kinetic rate law parameters on a $\mathrm{Na}-\mathrm{Ca}-\mathrm{Al}$ borosilicate glass for low-activity waste. J. Nucl. Mater. 249, 175-189 (1997).

11. Crovisier, J. L., Advocat, T. \& Dussossoy, J. L. Nature and role of natural alteration gels formed on the surface of ancient volcanic glasses (Natural analogs of waste containment glasses). J. Nucl. Mater. 321, 91-109 (2003).

12. Gin, S., Beaudoux, X., Angeli, F., Jegou, C. \& Godon, N. Effect of composition on the short-term and long-term dissolution rates of ten borosilicate glasses of increasing complexity from 3 to 30 oxides. J. Non-Cryst. Solids 358, 2559-2570 (2012).

13. Gin, S. et al. The fate of silicon during glass corrosion under alkaline conditions: a mechanistic and kinetic study with the international simple glass. Geochim. Cosmochim. Acta 151, 68-85 (2015).

14. Beaucaire, C. et al. A thermodynamic model for the prediction of pore water composition of clayey rock at 25 and 80 degrees $\mathrm{C}$-comparison with results from hydrothermal alteration experiments. Chem. Geol. 334, 62-76 (2012).

15. Inagaki, Y., Kikunaga, T., Idemitsu, K. \& Arima, T. Initial dissolution rate of the international simple glass as a function of $\mathrm{ph}$ and temperature measured using microchannel flow-through test method. Int. J. Appl. Glass Sci. 4, 317-327 (2013).

16. Lasaga, A. C., Soler, J. M., Ganor, J., Burch, T. E. \& Nagy, K. L. Chemicalweathering rate laws and global geochemical cycles. Geochim. Cosmochim. Acta 58, 2361-2386 (1994).

17. Grambow, B. A general rate equation for nuclear waste glass corrosion. Mater. Res. Soc. Symp. Proc. 12, 15-27 (1985).

18. Frugier, P. et al. SON68 nuclear glass dissolution kinetics: current state of knowledge and basis of the new GRAAL model. J. Nucl. Mater. 380, 8-21 (2008).

19. Daux, V., Guy, C., Advocat, T., Crovisier, J. L. \& Stille, P. Kinetic aspects of basaltic glass dissolution at 90 degrees C: role of aqueous silicon and aluminium. Chem. Geol. 142, 109-126 (1997).

20. Gin, S., Ryan, J. V., Schreiber, D. K., Neeway, J. \& Cabie, M. Contribution of atom-probe tomography to a better understanding of glass alteration mechanisms: application to a nuclear glass specimen altered 25 years in a granitic environment. Chem. Geol. 349, 99-109 (2013).

21. Bunker, B. C. molecular mechanisms for corrosion of silica and silicate-glasses. J. Non-Cryst. Solids 179, 300-308 (1994).

22. Casey, W. H. \& Bunker, B. Leaching of Mineral and glass surfaces during dissolution. Rev. Mineral. Geochem. 23, 397-426 (1990).

23. Angeli, F. et al. Influence of calcium on sodium aluminosilicate glass leaching behaviour. Phys. Chem. Glasses 42, 279-286 (2001).

24. Valle, N. et al. Elemental and isotopic $\left({ }^{29} \mathrm{Si}\right.$ and $\left.{ }_{18} \mathrm{O}\right)$ tracing of glass alteration mechanisms. Geochim. Cosmochim. Acta 74, 3412-3431 (2010).

25. Gin, S., Ribet, I. \& Couillard, M. Role and properties of the gel formed during nuclear glass alteration: importance of gel formation conditions. J. Nucl. Mater. 298, 1-10 (2001).

26. Xing, S. B., Buechele, A. C. \& Pegg, I. L. Effect of surface-layers on the dissolution of nuclear waste glasses. Mater. Res. Soc. Symp. Proc. 333, 541-548 (1994).

27. Delage, F., Ghaleb, D., Dussossoy, J. L., Chevallier, O. \& Vernaz, E. A mechanistic model for understanding nuclear waste glass dissolution. J. Nucl. Mater. 190, 191-197 (1992)

28. Daval, D. et al. Influence of amorphous silica layer formation on the dissolution rate of olivine at 90 degrees C and elevated pCO(2). Chem. Geol. 284, 193-209 (2011).

29. Wang, Y. F. Nanogeochemistry: nanostructures, emergent properties and their control on geochemical reactions and mass transfers. Chem. Geol. 378, 1-23 (2014).

30. Boksay, Z., Bouquet, G. \& Dobos, S. Kinetics of formation of leached layers on glass surfaces. Phys. Chem. Glasses 9, 69-71 (1968).

31. Lanford, W. A. et al. Hydration of soda-lime glass. J. Non-Cryst. Solids 33, 249-266 (1979).

32. Doremus, R. H. in Treatise on Materials Science Vol. 17 (eds Tomozawa, M. \& Doremus, R. H.) 41-69 (Academic Press, 1979).

33. Ledieu, A., Devreux, F., Barboux, P., Sicard, L. \& Spalla, O. Leaching of borosilicate glasses. I. Experiments. J. Non-Cryst. Solids 343, 3-12 (2004).

34. Angeli, F., Charpentier, T., Gin, S. \& Petit, J. C. O-17 3Q-MAS NMR characterization of a sodium aluminoborosilicate glass and its alteration gel. Chem. Phys. Lett. 341, 23-28 (2001)

35. Cho, H. et al. Solution state structure determination of silicate oligomers by Si-29 NMR spectroscopy and molecular modeling. J. Am. Chem. Soc. 128, 2324-2335 (2006)

36. Iler, R. K. The Chemistry of Silica (John Wiley \& Sons, 1979).

37. Engelhardt, G. \& Michel, D. High-Resolution Solid-State NMR of Silicates and Zeolites (John Wiley \& Sons, 1987).

38. Nanba, T., Nishimura, M. \& Miura, Y. A theoretical interpretation of the chemical shift of Si-29 NMR peaks in alkali borosilicate glasses. Geochim. Cosmochim. Acta 68, 5103-5111 (2004).
39. Soleilhavoup, A., Delaye, J. M., Angeli, F., Caurant, D. \& Charpentier, T. Contribution of first-principles calculations to multinuclear NMR analysis of borosilicate glasses. Magn. Reson. Chem. 48, S159-S170 (2010).

40. Angeli, F., Charpentier, T., de Ligny, D. \& Cailleteauz, C. Boron speciation in soda-lime borosilicate glasses containing zirconium. J. Am. Ceram. Soc. 93, 2693-2704 (2010).

41. Schneider, J. et al. $\mathrm{Q}(\mathrm{n})$ distribution in stoichiometric silicate glasses: thermodynamic calculations and Si-29 high resolution NMR measurements. J. Non-Cryst. Solids 325, 164-178 (2003).

42. Larsen, F. H. \& Farnan, I. Si-29 and O-17 (Q)CPMG-MAS solid-state NMR experiments as an optimum approach for half-integer nuclei having long T-1 relaxation times. Chem. Phys. Lett. 357, 403-408 (2002).

43. Dohmen, L. et al. Pattern Formation in Silicate Glass Corrosion Zones. Int. J. Appl. Glass Sci. 4, 357-370 (2013).

44. Geisler, T. et al. Aqueous corrosion of borosilicate glass under acidic conditions: a new corrosion mechanism. J. Non-Cryst. Solids 356, 1458-1465 (2010).

45. Hellmann, R. et al. Unifying natural and laboratory chemical weathering with interfacial dissolution-reprecipitation: a study based on the nanometer-scale chemistry of fluid-silicate interfaces. Chem. Geol. 294, 203-216 (2012).

46. Geisler, T., Schaltegger, U. \& Tomaschek, F. Re-equilibration of zircon in aqueous fluids and melts. Elements 3, 43-50 (2007).

47. McGrail, B. P. et al. The structure of Na2O-Al2O3-SiO2 glass: impact on sodium ion exchange in $\mathrm{H} 2 \mathrm{O}$ and D2O. J. Non-Cryst. Solids 296, 10-26 (2001)

48. Cailleteau, C. et al. Insight into silicate-glass corrosion mechanisms. Nat. Mater. 7, 978-983 (2008).

49. Jollivet, P. et al. Investigation of gel porosity clogging during glass leaching. J. Non-Cryst. Solids 354, 4952-4958 (2008).

50. Bourg, I. C. \& Steefel, C. I. Molecular dynamics simulations of water structure and diffusion in silica nanopores. J. Phys. Chem. C 116, 11556-11564 (2012).

51. Briman, I. M. et al. Impact of pore size and pore surface composition on the dynamics of confined water in highly ordered porous silica. J. Phys. Chem. C 116, 7021-7028 (2012)

52. Chamssedine, F., Sauvage, T., Peuget, S., Fares, T. \& Martin, G. Helium diffusion coefficient measurements in R7T7 nuclear glass by He-3(d,alpha)H-1 nuclear reaction analysis. J. Nucl. Mater. 400, 175-181 (2010).

53. Libourel, G. et al. The use of natural and archeological analogues for understanding the long-term behavior of nuclear glasses. C. R. Geosci. 343, 237-245 (2011).

54. Michelin, A. et al. Silicate glass alteration enhanced by iron: origin and longterm Implications. Environ. Sci. Technol. 47, 750-756 (2013).

55. Curti, E., Crovisier, J. L., Morvan, G. \& Karpoff, A. M. Long-term corrosion of two nuclear waste reference glasses (MW and SON68): a kinetic and mineral alteration study. Appl. Geochem. 21, 1152-1168 (2006).

56. Cunnane, J. C. et al. High-level nuclear-waste borosilicate glass-a compendium of characteristics. Mater. Res. Soc. Symp. Proc. 294, 225-232 (1993).

57. Bourcier, W. L., Peiffer, D. W., Knauss, K. G., Mckeegan, K. D. \& Smith, D. K. A kinetic-model for borosilicate glass dissolution based on the dissolution affinity of a surface alteration layer. Mater. Res. Soc. Symp. Proc. 176, 209-216 (1990).

58. Munier, I., Crovisier, J. L., Grambow, B., Fritz, B. \& Clement, A. Modelling the alteration gel composition of simplified borosilicate glasses by precipitation of an ideal solid solution in equilibrium with the leachant. J. Nucl. Mater. 324, 97-115 (2004).

59. Crank, J. The Mathematics of Diffusion (Oxford Science Publication, 1980).

60. Giannuzzi, L. A. \& Stevie, F. A. A review of focused ion beam milling techniques for TEM specimen preparation. Micron 30, 197-204 (1999).

61. Bonnet, N., Colliex, C., Mory, C. \& Tence, M. Developments in processing image sequences for elemental mapping. Scanning Microsc. 2, 351-364 (1988)

\section{Acknowledgements}

This work was supported by CEA and Areva. We are grateful to Yves Minet, Alex Mitroshkov, Laurent Dupuy, Claude Berthon, Martiane Cabié, Jean-Louis Chouchan, Céline Marcou, Jessica Vincent and Jean-Pierre Mestre for their technical support. We also thank Baptiste Gault, Lindsey Neill, Benjamin Parruzot and François Sudreau for their helpful comments.

\section{Author contributions}

S.G. supervised the study and wrote the paper. P.J. and M.F. were deeply involved in the interpretation. P.F. modeled the B profiles. F.A. and T.C. performed the solid-state NMR study.

\section{Additional information}

Supplementary Information accompanies this paper at http://www.nature.com/ naturecommunications 
Competing financial interests: The authors declare no competing financial interests.

Reprints and permission information is available online at http://npg.nature.com/ reprintsandpermissions/

How to cite this article: Gin, S. et al. Origin and consequences of silicate glass passivation by surface layers. Nat. Commun. 6:6360 doi: 10.1038/ncomms7360 (2015) (c) (i) This work is licensed under a Creative Commons Attribution 4.0 International License. The images or other third party material in this article are included in the article's Creative Commons license, unless indicated otherwise in the credit line; if the material is not included under the Creative Commons license, users will need to obtain permission from the license holder to reproduce the material. To view a copy of this license, visit http://creativecommons.org/licenses/by/4.0/ 